\title{
Assessment of Bacteriological Changes in Leprosy, Based on Serial Biopsies
}

\author{
D. L. LEIKER
}

\author{
Royal Tropical Institute, Amsterdam, The Netherlands
}

\begin{abstract}
After a discussion of some of the advantages and disadvantages of the smear technique in estimating the bacteriological changes that occur in patients with leprosy, the author then describes in some detail his method of assessing these changes by the examination of sections of serial biopsy specimens. Although the method, which is fully described, is difficult and cannot be regarded as ideal, he concludes nevertheless that the results are far superior to those of assessment based on smears.
\end{abstract}

\section{Introduction}

The bacteriological status of leprosy patients is usually assessed by taking smears. The advantages are that the technique is relatively simple, that sophisticated laboratory facilities are not required, and that several smears from different sites can be taken in one session without too much inconvenience to the patient. If, however, biopsies of a patient suffering from a paucibacillary form of leprosy (whose smears may not reveal any bacilli) are examined, bacilli are not infrequently found in the sections, occasionally in the infiltrates in the corium or subcutaneous tissues, but more frequently in nerve twigs, vessel walls, and in smooth muscle tissues. Such bacilli would rarely be present in the material obtained by the usual technique of taking smears.

It has also been found that the Bacterial Index (BI) in smears from one lesion may differ considerably when the smears are taken by different technicians, and even when smears from the same lesion are taken after a brief interval by the same technician. This observation should not cause surprise, since many variables may vitiate the results. For instance, the total number of bacilli observed depends on the actual amount of dermal tissue obtained and the area on the slide on which the material is spread. The latter can be standardized, but the amount of tissue taken may vary considerably. The latter depends on the length and the depth of the incision, on the number of times the incision is scraped, on the force used, on the shape and sharpness of the knife, and on the physical condition of the tissue. The latter varies from site to site and may change with time (e.g. owing to fibrosis or influenced by a reactional phase).

Variables in staining technique and in assessment of the BI and the Morphological Index (MI) may be avoided by sending the unstained smears to one investigator for processing and examination. Because of the variables in the taking of smears, however, the results of examination of smears from different centres do not appear to be comparable. Many of these disadvantages disappear, or at 
least decrease, if the assessment of the bacteriological changes is based on serial biopsies taken from the same lesion.

\section{Technique of Collecting Specimen}

A lesion sufficiently large to allow the taking of several biopsy specimens should be selected. If such a large lesion is not present, more than one lesion, all of the same age and clinically similar, should be selected. The successive biopsies are taken from a site adjacent to the first biopsy, just avoiding the scars of previous biopsies. The punch-biopsy method (using a 4- to 6-mm punch) has the advantage that the technique is simple and that several biopsies can be taken from one moderately large lesion. The punches should be sharp, otherwise a considerable part of the small specimen is seriously damaged. The specimen should include subcutaneous tissue and be handled at the outer edges only with a forceps with fine teeth. Fixation in $4 \%$ neutral formalin solution $(10$ times diluted commercial stock solution) is to be preferred to other more complicated fixation techniques because of the smaller chance of error. The risk of damage to the specimen and the costs of mailing (postage) are greatly reduced by using plastic containers (5-ml serum tubes with lid, sealed with tape).

In our laboratory, the Triff method is used for routine staining. Although this method gives excellent results in the hands of experienced technicians, the morphology of bacilli is less readily determined than in sections stained according to the Fite-Faraco-Wade technique. The assessment of bacteriological changes is therefore based on the latter.

One advantage of this method is that there are fewer variables in the taking of biopsy specimens than in taking skin smears; the results of examination of material from different centres by one examiner are thus more likely to be comparable. This consideration is important in view of the increasing shortage, in many centres, of patients suitable for drug trials. A second advantage is that the examination of successive biopsies by an independent assessor provides a check on the selection of patients for trial; in particular, patients exhibiting some borderline features can thereby be excluded from a trial designed to embrace only those with lepromatous leprosy.

\section{Technique of Assessment}

The Bacteriological Index is found by counting the numbers of bacilli in a number of fields of infiltrated parts of the section, if the BI is found to be below Grade 5+ (Ridley's decimal scale). The difference between Grade 5+ (100-1000 bacilli per field) and Grade $6+$ (more than 1000 bacilli per field) is estimated. Bacilli in globi are not counted, but estimated. Bacilli in bacilliferous macrophages are counted only if the separate bacilli can be clearly distinguished.

The decimal grading system proposed by Ridley has one disadvantage. The highest grade is $6+$, meaning 1000 or more bacilli per field. Frequently, the number of bacilli in sections is 10 , or even more, times higher than 1000 per field. The difference between 10000 and 1000 per field is not recorded, and changes in the BI are not apparent until the number of bacilli has decreased to less than 1000 per field. Because the elimination of dead bacilli is a slow process in patients with lepromatous leprosy, one or more years may elapse before any decrease in the BI becomes apparent. Because the number of bacilli in smears is on the average 
smaller than in histological sections, this disadvantage is less serious if the BI is based on smears. It may be useful to extend the decimal scale to 10+ (Gaffky), although the grades above 5+ can only be estimated.

The Granularity Index (percentage of granular bacilli) is found by examining the morphology of 100 to 200 bacilli, excluding those in globi, but including those in bacilliferous histiocytes if the individual bacilli can be clearly distinguished.

Because it is more difficult to determine the morphology of the bacilli in sections as compared with smears, 3 categories of bacilli are distinguished: (1) Bacilli that are completely and evenly stained are recorded as intact (I); they are regarded as viable. (2) Bacilli that are somewhat unevenly stained, but are not yet granular, are recorded as fragmented $(\mathrm{F})$; this is seen to be a mixed group of viable and non-viable bacilli, since growth in the mouse footpad has been obtained from biopsy specimens containing no intact bacilli but about $10 \%$ fragmented bacilli. (3). Bacilli that appear as granules are recorded as granular $(\mathrm{G})$; such bacilli are regarded as non-viable. Actual counts are made of the percentages of the 3 categories of bacilli, using a tabulator similar to those used for differential counts of white blood cells. The Morphological Index corresponds to some extent, but not completely, with the total of intact and fragmented bacilli. In order to express the bacteriological status of the patient in one figure, the Granularity Index is preferred.

\section{Discussion}

Because it is more difficult to assess the BI and the morphology of the bacilli on sections as compared with smears, the method cannot be regarded as an ideal one. In our hands, however, the method was found to be far superior to the assessment based on smears alone. It has been found that a technician is able to achieve uniform and dependable results after some months of experience. Control is by counting blindly a number of sections for a second time after an interval of a week or two. The results of a second technician were not accepted for trial work until his counts in a series of sections were comparable with those of the first technician. Unexpected changes in the MI or the morphology of the bacilli were always checked; with few exceptions, the findings of a trained technician were confirmed. The results of blind assessment of thousands of sections in the past years have been very consistent, which is regarded as evidence of the relative reliability of the method.

It has been found that the proportion of completely intact bacilli is higher in smears as compared with sections of the same lesion from the same patient. It is probable that the staining properties of the bacilli are affected by the more elaborate processing technique of preparing sections. Because the error is constant and in the same sense, the reliability of the method for assessing bacteriological changes is not impugned.

It has been objected that valid conclusions cannot be drawn from the examination of only one biopsy specimen. And it is admitted that the BI in lesions of different age or from different sites of the body may indeed differ significantly.

In this respect a series of smears undoubtedly gives a more accurate picture of the BI. This disadvantage may to some extent be reduced by selecting a fairly advanced lesion for biopsy examination. Much more important than the BI is 
usually the morphology of the bacilli. The examination of one biopsy specimen gives a reasonably accurate picture of the morphological status of the bacilli of the whole patient, since the changes in bacillary morphology in the various lesions of the skin and the mucosa are very similar. There is one exception however. The pattern may be different in different lesions of patients during an exacerbation or a relapse of the disease, for example, due to the development of drug resistance.

In spite of some disadvantages, the method described is regarded as the most satisfactory for the assessment of the bacteriological effect of treatment, and in particular for drug trials. 